\title{
Orbit correction in a linear nonscaling fixed field alternating gradient accelerator
}

\author{
D. J. Kelliher ${ }^{*}$ and S. Machida \\ ASTeC, STFC Rutherford Appleton Laboratory, Harwell Oxford, Didcot, \\ Oxfordshire OX11 OQX, United Kingdom \\ C. S. Edmonds and I. W. Kirkman \\ University of Liverpool, Liverpool L69 7ZE, United Kingdom \\ and Cockcroft Institute of Accelerator Science and Technology, \\ Daresbury, Warrington WA4 4AD, United Kingdom \\ J. K. Jones and B. D. Muratori \\ ASTeC, STFC Daresbury Laboratory, Daresbury, Warrington WA4 4AD, United Kingdom \\ and Cockcroft Institute of Accelerator Science and Technology, \\ Daresbury, Warrington WA4 4AD, United Kingdom \\ J. M. Garland \\ University of Manchester, Manchester M13 9PL, United Kingdom \\ and Cockcroft Institute of Accelerator Science and Technology, \\ Daresbury, Warrington WA4 4AD, United Kingdom

$$
\text { J.S. Berg }{ }^{\dagger}
$$ \\ Brookhaven National Laboratory, Upton, New York 11973-5000, USA
}

(Received 28 July 2014; published 20 November 2014)

\begin{abstract}
In a linear nonscaling fixed field alternating gradient accelerator, the large natural chromaticity of the machine results in a betatron tune that varies by several integers over the momentum range. Orbit correction is complicated by the consequent variation of the phase advance between lattice elements. Here we investigate how the correction of multiple closed orbit harmonics allows correction of both the closed orbit distortion and the accelerated orbit distortion over the momentum range.
\end{abstract}

DOI: 10.1103/PhysRevSTAB.17.112806

PACS numbers: 29.20.-c, 29.27.-a

\section{INTRODUCTION}

The linear nonscaling fixed field alternating gradient accelerator (linear NS-FFAG) is proposed as a candidate for muon acceleration in facilities such as a neutrino factory [1] or muon collider [2], as a compact device for hadron therapy [3] and as an electron driver for an electron-ion collider [4]. Very rapid acceleration, a requirement in the case of short-lived muons, is enabled both by the fact that the fields of the magnets are fixed and by the use of the

\footnotetext{
*david.kelliher@stfc.ac.uk

This manuscript has been coauthored by an employee of Brookhaven Science Associates, LLC under Contract No. DEAC02-98CH10886 with the U.S. Department of Energy. The United States Government retains a nonexclusive, paid-up, irrevocable, worldwide license to publish or reproduce the published form of this manuscript, or allow others to do so, for United States Government purposes.

Published by the American Physical Society under the terms of the Creative Commons Attribution 3.0 License. Further distribution of this work must maintain attribution to the author $(s)$ and the published article's title, journal citation, and DOI.
}

serpentine channel for acceleration [5]. The absence of nonlinearity in the magnet field profile results in the large dynamic aperture required to accommodate a muon beam. The linear NS-FFAG has in recent years been under investigation using EMMA (Electron Model for Many Applications), a prototype built and commissioned at Daresbury Laboratory, United Kingdom. Acceleration in the serpentine channel [6] and a large dynamic aperture [7] have been demonstrated in this machine. Here we develop methods to correct the closed and accelerated orbit in this novel type of accelerator and report on EMMA experimental results.

Since EMMA consists of linear elements only, the betatron tune declines with momentum according to the natural chromaticity of the machine. Over the $10-20 \mathrm{MeV} / c$ momentum range of the machine, the ring tune passes through several integers in the horizontal and vertical planes. In general, correction of the closed orbit distortion (COD) at a single momentum does not ensure that the orbit is corrected at other momenta where the tune is significantly different. Instead, to correct both the COD 
and the accelerated orbit, the magnet-lattice-error harmonics associated with each integer tune over the momentum range should be corrected.

The paper begins by describing the experimental setup in EMMA (Sec. II). The theory of harmonic correction of the COD and as an alternative, a modified least-squares correction, is described in Sec. III. Harmonic correction of the accelerated orbit distortion is investigated by simulation in Sec. IV. The methodology used to measure the closed orbit and orbit response matrix is described (Sec. V) before the experimental results are presented (Sec. VI). Some limitations of the correction method and the implications of the findings of this paper are discussed in Sec. VII.

\section{EXPERIMENTAL SETUP}

EMMA is a ring consisting of 42 quadrupole doublet pairs each separated by a $21-\mathrm{cm}-$ long drift. The quadrupoles are displaced horizontally to supply the bend angle required. As can be seen in Fig. 1, the elements in each cell are collinear with a $2 \pi / 42$ rotation between neighboring cells at the entrance of the $D$ quadrupole magnet. Diagnostics include two turn-by-turn four-button beam position monitors (BPMs) in most cells; there is a BPM in the short drift between each quadrupole doublet and two at either end of alternate long drifts (apart from in the injection and extraction regions). Each quadrupole is mounted on a slider that allows the magnet's horizontal position to be changed remotely. This feature was included in the first instance to allow various lattice configurations to

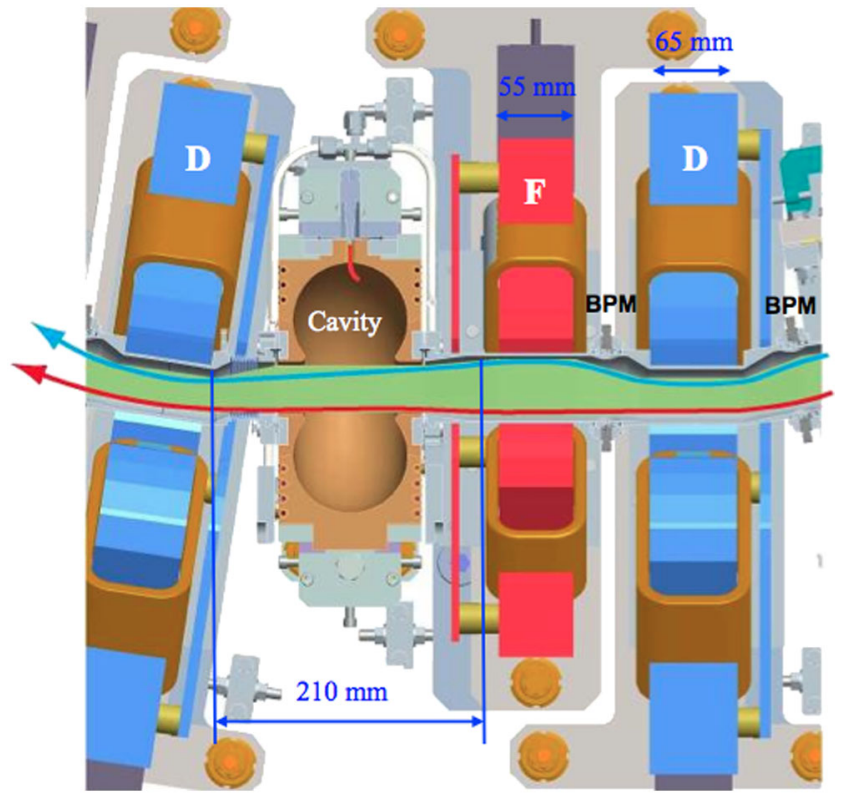

FIG. 1. Schematic of an EMMA cell showing a $D$ (blue) and $F$ (red) quadrupole doublet, rf cavity, and BPMs (dark gray). The blue and red arrows indicate the paths taken by the low and high momentum electrons, respectively. be selected but also to facilitate orbit correction in the horizontal plane. There are 16 vertical correctors distributed around the ring. Apart from in the injection and extraction region, these vertical correctors are located in every other cell. More details about the experimental setup can be found in Ref. [8].

Since there is, by design, a horizontal excursion ( $\sim 20 \mathrm{~mm})$ with momentum in EMMA, the BPMs must be able to cope with a bunch that moves across a significant fraction of its 48-mm aperture. A 3D electrostatic model of the BPM allows voltage ratios to be mapped to coordinates across the aperture range [9].

For ease of operation, the so-called "equivalent momentum" approach was adopted when scanning the fixed momentum range. This entailed scaling the quadrupole gradient (while keeping the same $D / F$ gradient ratio) rather than changing the momentum of the injected beam [6]. The lattice parameters will be the same whether the real or equivalent momentum is varied, so long as the magnets are far from saturation. However, the time of flight must be scaled by the ratio of beam velocity difference between the real and equivalent momenta.

\section{CORRECTION OF COD ACROSS MULTIPLE MOMENTA}

Expressed in terms of Fourier harmonics, the closed orbit distortion $y_{\mathrm{co}}$ resulting from all error harmonics may be written

$$
y_{\mathrm{co}}(s)=\sqrt{\beta(s)} \sum_{k=-\infty}^{\infty} \frac{q^{2} f_{k}}{q^{2}-k^{2}} e^{j k \phi}
$$

where $q$ is the tune, $k$ is the harmonic number, $\beta$ is the betatron function, and $\phi$ is the phase advance. The Fourier excitation strength $f_{k}$ is driven by the dipole errors $\Delta B$ distributed around the ring and is given by

$$
f_{k}=\frac{1}{2 \pi q} \oint \sqrt{\beta} \frac{\Delta B}{B \rho} e^{-j k \phi} d s,
$$

where $B \rho$ is the magnetic rigidity. Note that, since the Fourier function is Hermitian, it follows $f_{-k}=\bar{f}_{k}$. From Eqs. (1) and (2), and by neglecting the variation in $\beta$, it can be seen that the amplitude of any harmonic component $k$ of the COD decreases with $\left|q^{2} /\left(q^{2}-k^{2}\right)\right|$. It follows that the COD at any particular momentum is dominated by the harmonics associated with the nearby integer tunes. Furthermore, it is clear that correcting for a single harmonic should, in principle, allow us to eliminate that component of the COD while having no effect on the other harmonicsprovided that the character of the normalized lattice errors $\Delta B / B$ is independent of the radius across the relatively large aperture. A set of harmonic corrections may be added allowing the COD across the momentum range to be corrected. 
Note that, in a FFAG, the synchrotron concept of a "reference" or "golden" orbit which serves as the goal for orbit correction is not so practical; it is difficult to establish the ideal closed orbit at any momentum by simulation with any confidence, given the complexities of modeling offaxis trajectories in quadrupole doublet magnets with overlapping fringe fields. Instead, the goal is to minimize the variation in closed orbit measured at BPMs distributed around the ring about the mean at each momentum. Since we make use of BPMs located at the same position in each of the 42 identical cells, they should all record the same position at each momentum if there is no orbit distortion.

In the harmonic correction scheme, the set of corrections follows the pattern of the harmonics closest to the betatron tune. To correct the $k$ th harmonic, a set of $N_{C}$ dipole correctors are powered to achieve angular kicks:

$$
\theta_{j}=\frac{1}{\sqrt{\beta_{j}}}\left(a_{k} \cos k \phi_{j}+b_{k} \sin k \phi_{j}\right) \quad\left(j=1, \ldots, N_{c}\right),
$$

where $\beta_{j}$ and $\phi_{j}$ are the betatron amplitude and phase at the corrector locations [10], respectively, and the coefficients $a_{k}$ and $b_{k}$ are chosen to match the phase and amplitude of the harmonic in question. Since a linear NS-FFAG has a high degree of symmetry, it is reasonable to assume, in the first instance, a uniform phase advance per cell and a constant value of $\beta_{j}$ at the corrector locations.

In that case, Eq. (3) may be rewritten as follows:

$$
\theta_{j}=\frac{1}{\sqrt{\beta_{c}}}\left(a_{k} \cos \frac{2 \pi k j}{N_{c}}+b_{k} \sin \frac{2 \pi k j}{N_{c}}\right) \quad\left(j=1, \ldots, N_{c}\right) .
$$

The orbit response matrix $A$, calculated by measuring the change in the closed orbit when each corrector in turn is excited, can be used to choose $a_{k}$ and $b_{k}$ coefficients in Eq. (4) to cancel the phase and amplitude of the COD harmonic in question.

In the case where the closed orbit measurements are ideal, it is possible to obtain the harmonic content of any integer $k$ from the FFT of measurements made at any momentum ${ }^{1}$ no matter how different it is in term of tune $q$. However, as the amplitude of any harmonic component $k$ decreases with an increasing difference in tune, the effect of closed orbit measurement errors increasingly reduces the accuracy of the harmonic content calculation. In particular, BPM offsets may introduce spurious harmonics that mask the phase and amplitude of the underlying error sources. For that reason, the harmonic content should be determined by measuring the closed orbit where the tune $q$ as is close to

\footnotetext{
${ }^{1}$ Provided the character of errors $\Delta B / B$ does not vary across the aperture.
}
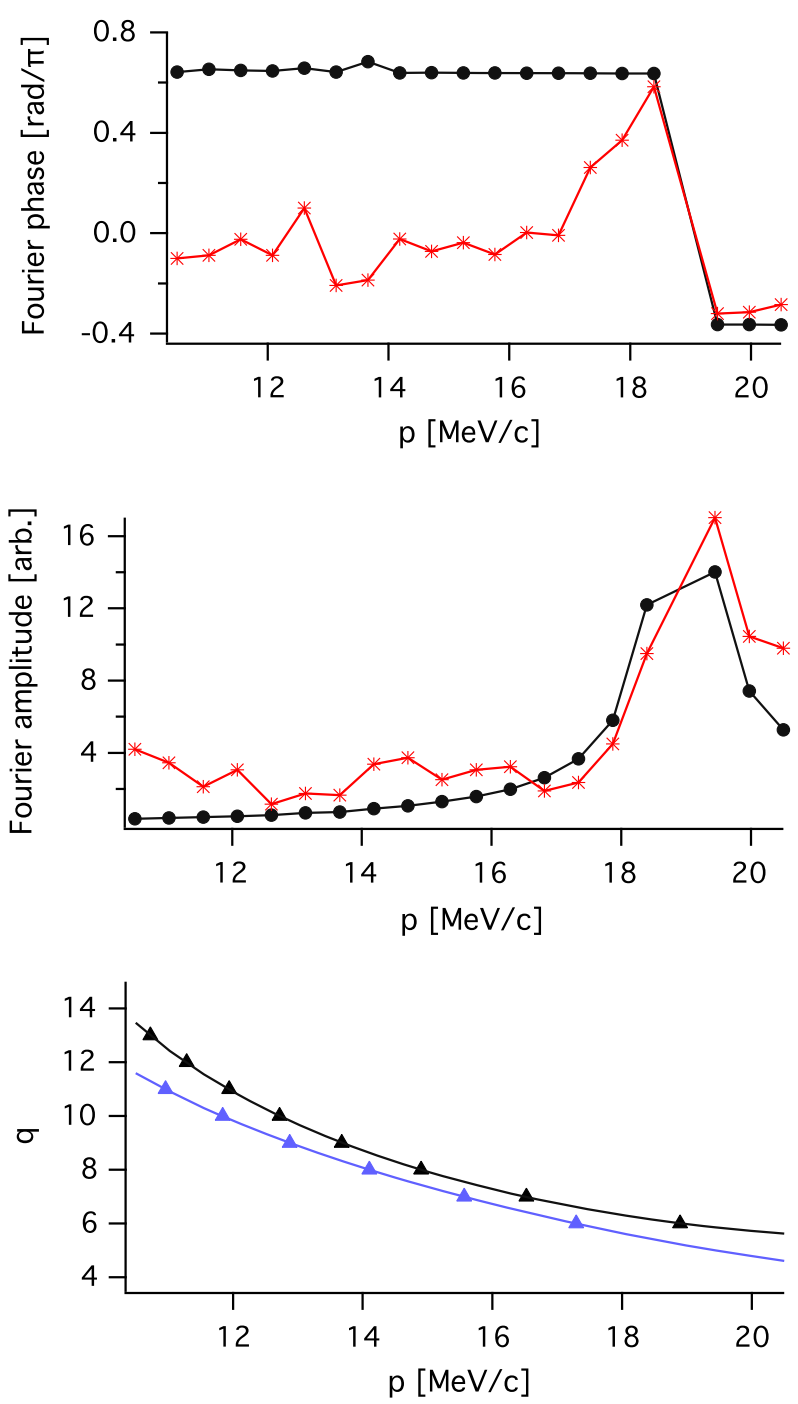

FIG. 2. Simulated harmonic six content of the horizontal COD showing the Fourier phase (top) and amplitude (middle) in the case where the closed orbit measurements are ideal (black circles) and where errors are included (red stars). Imperfect measurements are introduced by including BPM offsets $(\sigma=0.7 \mathrm{~mm})$ and an additional statistical error $(\sigma=0.2 \mathrm{~mm})$. The former is fixed for a given BPM, while the latter varies with every data point taken. The phase and amplitude for each Fourier component $z$ is given by $\tan ^{-1}[\operatorname{Im}(z) / \operatorname{Re}(z)]$ and $|z|$, respectively. Note that the Fourier amplitude is proportional to $q^{2} f_{k} /\left(q^{2}-k^{2}\right)$. The lower figure shows the simulated horizontal (black) and vertical (blue) tunes with the triangles indicating the integers. The example assumes magnet misalignments with standard deviation $0.1 \mathrm{~mm}$. These parameters were chosen to approximate those in the real machine.

$k$ possible. Figure 2 shows how the accuracy of the harmonic content calculation is affected by the magnitude of the BPM offsets.

The correction may be applied for a few iterations to make further improvements in the COD. At each step, as the COD is reduced, the effect of the BPM errors, which 
remain fixed in magnitude, on the harmonic content calculation at a particular momentum increases. Contrarily, a lower COD allows injection at momenta closer to the integer tune and so a more accurate determination of harmonic content.

For clarity, the correction scheme described above may be encapsulated in the following statements:

(i) Any and call closed orbits at any and all momenta are equally good reference orbits.

(ii) If $\Delta B / B$ does not vary substantially across the aperture, then the entire harmonic content of errors (i.e., the set $f_{k}$ ) may be extracted from the COD measured at any momentum-if measurements are ideal (i.e., error-free).

(iii) However, measurements are not ideal; and the inferred harmonic content may be corrupted.

(iv) The relative impact of measurement errors is least when the COD is large.

(v) The COD is large when the ring tune is close to a particular harmonic.

(vi) The complement to (v) is that the COD at a particular momentum is dominated by the field error harmonic(s) close to the tune for that momentum.

(vii) The COD at any and all momenta is reduced by implementing a correction of any and each harmonic excitation component $f_{k}$ [provided (ii) above is true].

(viii) Therefore, the recipe for orbit correction is to extract and correct harmonic excitations $f_{k}$ according to the procedure

(a) Set the momentum such that the ring tune $q$ is close to harmonic $k$ but COD is still well within the aperture.

(b) Measure COD and extract $f_{k}$.

(c) Correct $f_{k}$.

(d) Iterate steps (a)-(c) with $q$ closer to $k$.

(e) Repeat steps (a)-(d) for each integer $k$ within the range of $q$.

The COD is simulated by using the ZGOUBI tracking code [11]. The model of EMMA in the code is set up to approximately reproduce the measured tunes. In the example shown in Fig. 3, the COD is corrected by following the above algorithm. To replicate experimental uncertainties, the simulation includes fixed BPM offsets and statistical errors. The harmonic content associated with measurements near integer tunes 6-13 is used to correct the COD in that interval. As is done experimentally in the horizontal plane, the correction involved moving all $42 \mathrm{D}$ quadrupoles horizontally (each with a finite error). The figure shows that the COD is reduced over the momentum range of EMMA.

A weighted least-squares approach may also be considered to correct the COD at multiple momenta. In the standard single momentum case, $m$ COD measurements are corrected by using $n$ correctors making use of the $m \times n$ response matrix $A$. In this case, we extend the correction to cover $n p$ momenta by solving for $n p * m$ COD measurements, $y^{\text {ext }}$, using an extended $n p * m \times n$ response matrix $A^{\text {ext }}$. The least-squares correction seeks to minimize the

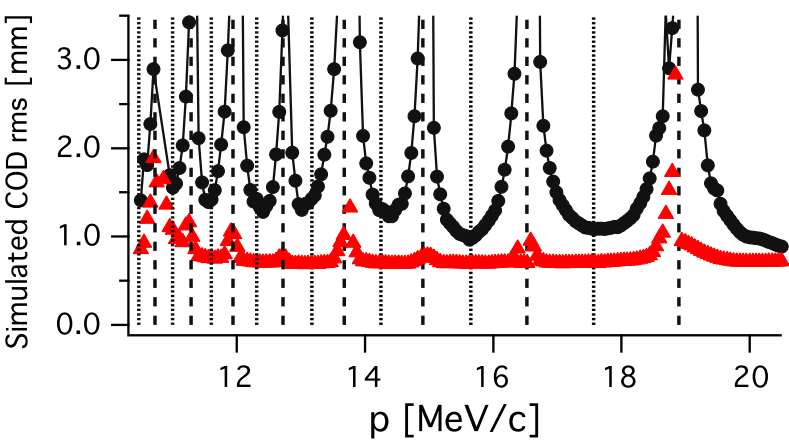

FIG. 3. Example of correction of multiple harmonics of the COD in the horizontal plane. From left to right, the eight peaks are associated with integer resonances 13-6. At each momentum, the closed orbit is found at simulated BPMs located in between each quadrupole doublet in the presence of magnet misalignments with standard deviation $0.1 \mathrm{~mm}$. Since the $42 \mathrm{BPMs}$ are symmetrically located in each cell, the closed orbit is identical in the case of an ideal lattice. Imperfect measurements are introduced by including BPM offsets $(\sigma=0.7 \mathrm{~mm})$ and an additional statistical error $(\sigma=0.2 \mathrm{~mm})$. The former is fixed for a given BPM, while the latter varies with every data point taken. These parameters were chosen to approximate those in the real machine. The rms of the resulting COD is then calculated at each momentum. The black circles and red triangles show this quantity before and after correction, respectively. Harmonics 6-13 are corrected, by following the method described in the algorithm, since they are within the tune range of EMMA. The correction is applied by moving the $D$ quadrupoles horizontally with finite error $(10 \mu \mathrm{m})$. The momenta at which the horizontal tune is integer and half-integer is shown by the vertical dashed and dotted lines, respectively.

norm of the extended data set $\left|y^{\mathrm{ext}}-A^{\mathrm{ext}} \theta\right|$. The amplitude of the correction can be adjusted by adding a $n \times n$ diagonal matrix to $A$ and padding the measurement vector $y_{\text {co }}$ with $n$ zeros. The diagonal elements can be adjusted to weight the amplitude of the resulting correction.

By selecting momenta in between different integer tunes, the correction settings will be dominated by the associated harmonics. However, in this case, the correction settings will also contain harmonics beyond the EMMA tune range caused by the finite accuracy of the closed orbit measurements. These spurious harmonics have a minimal impact on the COD but may increase the amplitude of the applied correction unnecessarily.

\section{HARMONIC CORRECTION OF ACCELERATED ORBIT DISTORTION}

The accelerated bunch receives a series of one or more transverse kicks that add coherently when a bunch passes through integer tunes excited by the corresponding harmonic of the error sources. In the case of a bunch with small amplitude initially (small in comparison with the effect of the kick), the magnitude of distortion after crossing is independent of phase. On the other hand, for a bunch with 
finite amplitude, the distortion may increase or decrease depending on its phase relative to the harmonic. Thus, the amplitude of an accelerated bunch will increase or decrease as it passes through multiple integer tunes.

When acceleration is sufficiently fast, the effect of the individual coherent kicks might not be apparent in the measurements. In Fig. 4, a single particle is injected with zero amplitude at $10.5 \mathrm{MeV} / c$ and accelerated in the serpentine channel to $20.5 \mathrm{MeV} / c$. In the figure, it is clear that, when the error pattern is excited with a single harmonic, the accelerated distortion increases when the momentum, and hence the betatron tune, of the particle crosses the associated integer. When all harmonics of the error sources are included, the resulting distortion is made up of a series of such coherent excitations as the particle passes through each integer tune in turn. However, the effect of each individual coherent excitation is not apparent in the distortion pattern.

The harmonic correction settings used to correct the closed orbit distortion will also, in principle, reduce the coherent excitations of the accelerated orbit. To illustrate this, we apply the same error sources and correction settings used to correct the closed orbit in Fig. 3. As before, acceleration is from 10.5 to $20.5 \mathrm{MeV} / c$ in the serpentine channel with 1.1 MV rf per turn. In Fig. 5, it is evident that the COD correction reduces the distortion over the momentum range.

It should be noted that correcting some subset of the harmonics does not always result in a reduction of the amplitude of the coherent excitation over the corresponding

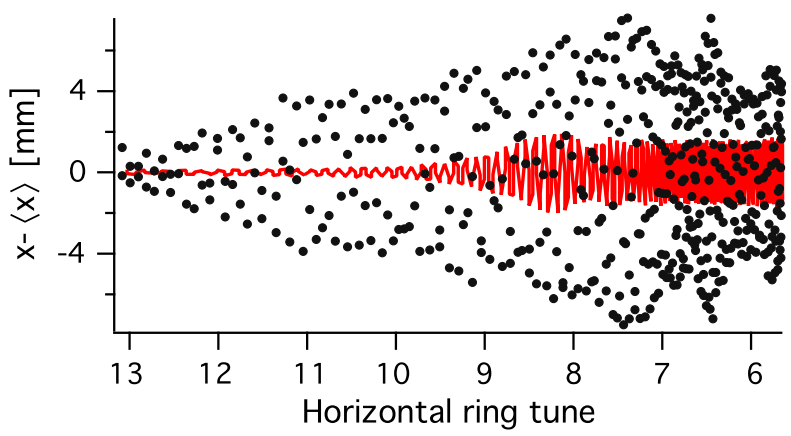

FIG. 4. Simulated distortion of the horizontal accelerated orbit versus tune in the case of where the magnets are misaligned randomly (black points) and in the case where just the ninth harmonic of the same random misalignment is retained (red line). The random misalignments have $50 \mu \mathrm{m}$ standard deviation. A single particle is tracked, starting on the closed orbit at the initial momentum, and its coordinate found throughout acceleration at simulated BPMs located in between each quadrupole doublet. The distortion is then calculated by subtracting from the coordinate the moving window average $\langle x\rangle$, with the width of the window determined by the betatron oscillation wavelength. In this case, the simulated BPM readings are assumed to be ideal. Acceleration is from 10.5 to $20.5 \mathrm{MeV} / c$ in the serpentine channel with 1.1 MV rf per turn.

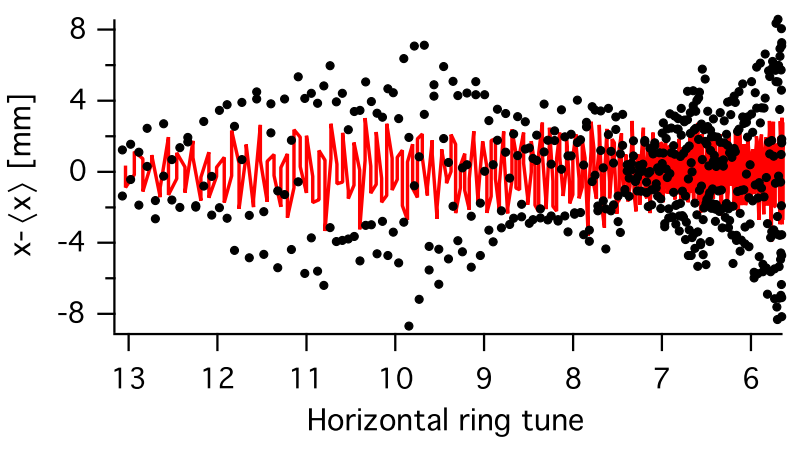

FIG. 5. Simulated accelerated orbit distortion versus tune before (black dots) and after (red line) COD correction of harmonics 6-13. The error sources and correction are as described for Fig. 3.

momentum range. As described above, the effect of an integer tune crossing may be to reduce the distortion; correcting the corresponding harmonic will remove this reduction.

\section{MEASUREMENT OF CLOSED ORBIT AND RESPONSE MATRIX}

The turn-by-turn coordinates obtained from the BPMs measure, for each turn, the center of mass of the bunch. Owing to the momentum spread of the beam and the lattice chromaticity, the betatron oscillations decohere, typically in a few tens of turns. Once the signal has fully decohered, the signal from the BPM in subsequent turns measures the closed orbit rather than the betatron oscillation.

In order to obtain a mean closed orbit at each BPM, a linear fit is made to the turn-by-turn coordinates. The fit excludes data from the first turn, which tends to be an outlier because of kicker jitter [8]. For turns after decoherence, coordinates whose difference from the fitted line is greater than 3 standard deviations are removed. The linear fit is then repeated and any outliers again removed. This procedure proceeds iteratively until no outliers remain. The nonzero slope of the linear fit, as seen in Fig. 6, may be the result of energy loss caused by beam loading [12]. The closed orbit is assumed to be the value of the linear fit at the first turn.

The response matrix is measured by applying a positive and negative transverse kick at each corrector in turn. The difference between the two closed orbit measurements yields the response. The magnitude of the kick should be large enough that the resulting closed orbit response is not dominated by measurement error, yet not so large as to lead to increased beam loss. It was determined experimentally that a reasonable response was obtained by horizontally moving the quadrupoles by $\pm 0.5 \mathrm{~mm}$ and applying \pm 0.2 A to the vertical correctors, resulting in a $\sim 3 \mathrm{mrad}$ and $\sim 1 \mathrm{mrad}$ kick in the horizontal and vertical planes, respectively. 


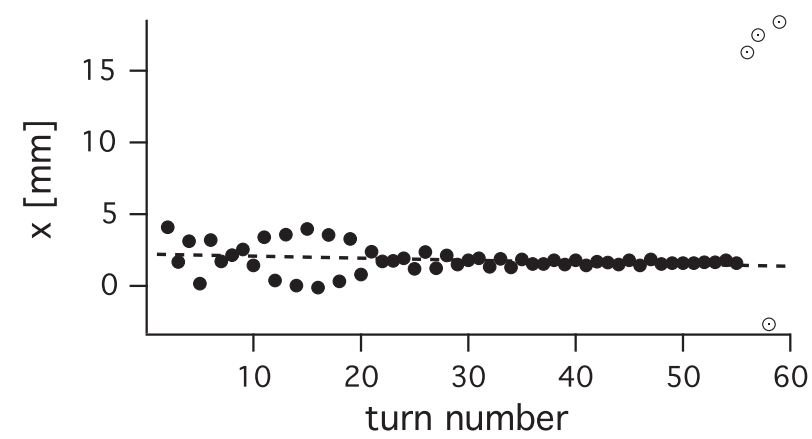

FIG. 6. Example turn-by-turn horizontal coordinate measured by a single BPM. The closed orbit is calculated by doing a linear fit to the coordinates (dashed line). Coordinates more than $3 \sigma$ from the mean are ignored by the fit (open circles).

To reduce the effect of errors and missing data in the response matrix measurement, it is desirable to fit an idealized response function to the data. The closed orbit at the $i$ th BPM $y_{i}$ owing to a kick $\theta_{j}$ at the $j$ th corrector is given by

$$
y_{i}=R_{i j} \theta_{j}
$$

where

$$
R_{i j}=\frac{\sqrt{\beta_{i} \beta_{j}}}{2 \sin (\pi q)} \cos \left(\left|\psi_{i}-\psi_{j}\right|-\pi q\right),
$$

where $q$ is the total betatron tune, $\beta_{i}$ and $\beta_{j}$ are the betatron function, and $\psi_{i}$ and $\psi_{j}$ are the phase advance at the BPM and corrector, respectively. Given the high degree of symmetry in EMMA, it is reasonable to assume the

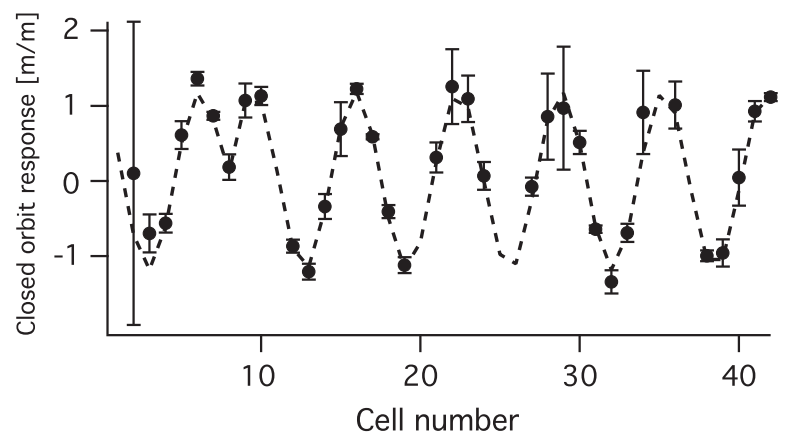

FIG. 7. Measurement of the response matrix generated by moving the $D$ quadrupole horizontally in cell 8 . The response is the difference of two closed orbits, measured with the quadrupole at $\pm 0.5 \mathrm{~mm}$, divided by the magnet shift. The error bars are obtained by adding in quadrature the standard deviation of the shot-by-shot variation of the two closed orbits. The dashed line shows the fit after optimizing the parameters in Eq. (5). In this case, the momentum is $18 \mathrm{MeV} / c$, the optimization finds the tune is 6.52 , the product of the betatron functions is $0.08 \mathrm{~m}^{2}$, and the phase difference between a $D$ quadrupole and its neighboring $\mathrm{BPM}$ is 0.16 rad. betatron functions and the phase advance between the corrector and BPM do not vary from cell to cell.

We best fit the $y_{i}$ at all BPMs to three parameters: the product $\sqrt{\beta_{i} \beta_{j}}$, the phase difference $\psi_{i}-\psi_{j}$, and the tune $q$. In order to make the optimization robust, a two-step procedure is employed. In the first step, the total tune is varied in small steps (0.01) over the expected integer range while the other parameters are fixed; the tune that results in the best fit to the data is found. In the second step, by using the tune found in the first step as a starting point, all parameters in Eq. (6) are optimized by using a downhill simplex algorithm. In both steps, the data to be fitted are weighted according to the standard deviation of closed orbit
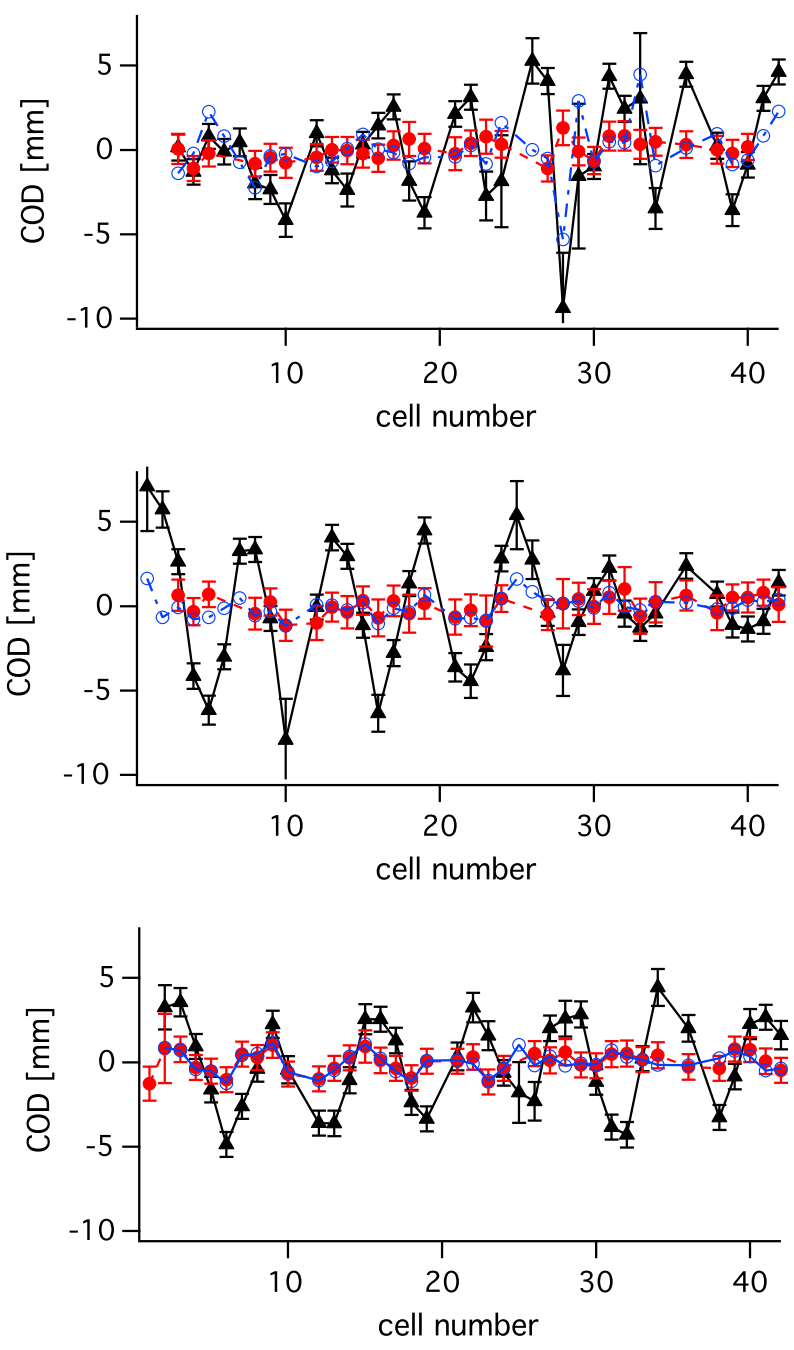

FIG. 8. Horizontal closed orbit correction at 14.3 (top), 16.1 (middle), and $18.0 \mathrm{MeV} / c$ (bottom) where the horizontal tunes are approximately $8.4,7.5$, and 6.5 , respectively. The closed orbit distortion measured before (black triangles) and after correction (red filled circles) is shown along with the predicted correction based on the response matrix (blue open circles). Error bars include the standard deviation of the shot-by-shot variation of the closed orbit as well as the BPM offset based on survey measurements. 
measurements. Note from Eq. (6) that this method finds the product of the betatron functions at the BPM and corrector rather than the individual values. An example measured response to a single corrector and the fit found by the algorithm is shown in Fig. 7. By repeating this calculation at several momenta and fitting the parameters by interpolating functions, the response matrix can be evaluated at all intervening momenta.

\section{EXPERIMENTAL RESULTS}

A set of closed orbit measurements was made across the momentum range in EMMA. A substantial closed orbit distortion was found ( $\sim 10 \mathrm{~mm}$ peak to trough) in both transverse planes. This is much greater than what would be expected from the surveyed magnet misalignments ( $\sim 0.1 \mathrm{~mm}$ peak to trough). The septum stray field has been identified as the major source in the horizontal plane, while in the vertical plane it has not yet been found.

The response matrix was measured at various momenta as described in Sec. V. A single least-squares correction (as described in Sec. III) involving three equivalent momenta
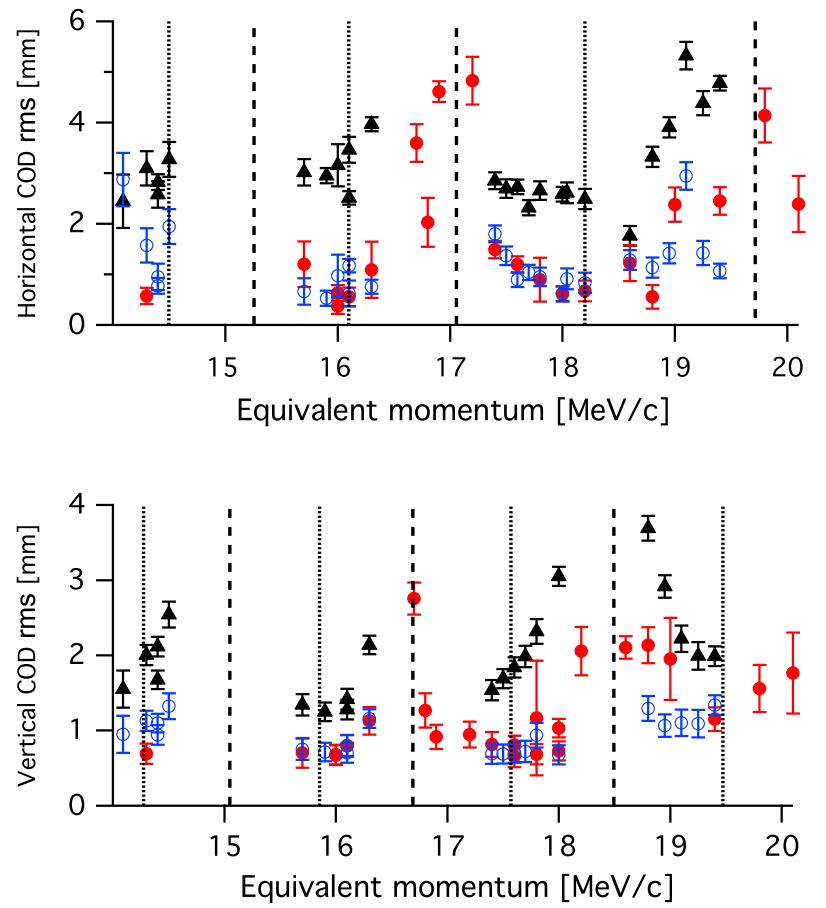

FIG. 9. The rms of COD over the momentum range in EMMA measured before (black triangles) and after (red filled circles) correction in the horizontal (top panel) and vertical (bottom panel) planes. At each momentum, the closed orbit is found at BPMs symmetrically located in between each quadrupole doublet and the rms of the resulting COD calculated. The predicted COD is also shown (blue open circles). The momenta at which the tune is integer and half integer, in the corresponding transverse plane, is shown by the vertical dashed and dotted lines, respectively. These values are obtained by fitting the tunes measured at various points over the momentum range by an interpolating function.
(14.3, 16.1, and $18 \mathrm{MeV} / c$ ), between consecutive pairs of integer tunes, was then applied in both transverse planes and the resulting closed orbits measured. The result of the correction at these three momenta is shown in Fig. 8. The correction achieved is broadly in agreement with the response matrix predictions. Maintaining the same correction settings, the COD was then measured over the entire momentum range. This enables to confirm the prediction (Sec. III) that COD correction at several particular momenta leads to compensation across a broad momentum range. In Fig. 9, the effect of the correction over the momentum range is presented-the COD is reduced and measurements can be made at momenta closer to integer tunes than before (for example, in the vicinity of $17 \mathrm{MeV} / c$, where the horizontal tune is close to 7). It can also be seen in both Figs. 8 and 9 that the predicted COD after correction is in broad agreement with the measurements.

Although harmonic correction as discussed in the previous section was not directly applied in the experiment, the correction found by least squares has the effect of reducing the dominant harmonics. In Fig. 10, the reduction in harmonics measured at a single momentum is shown. In Fig. 11, it can be seen that the amplitude of the harmonic 7 component of the COD is reduced across the momentum range. As in the simulation result (Fig. 2), the $\pi$ step change in phase on either side of the integer tune is apparent. The increased scatter in the harmonic phase data is consistent with the relatively increased effect of fixed measurement
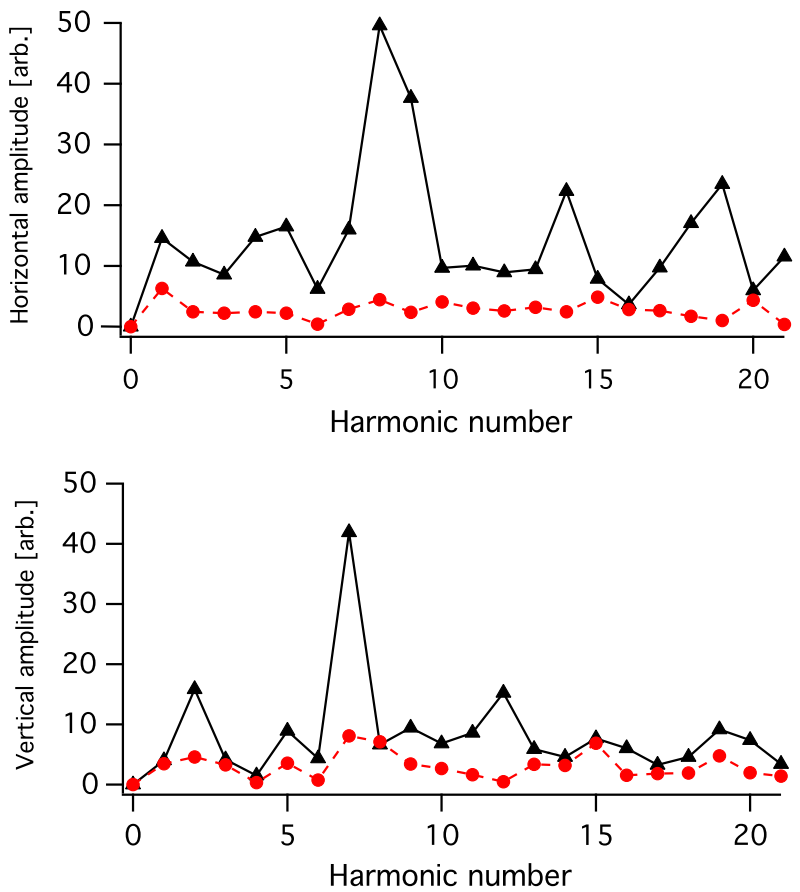

FIG. 10. Harmonics of the horizontal COD measured at 14.3 MeV/c before (black triangles) and after (red dots) correction in both transverse planes. 

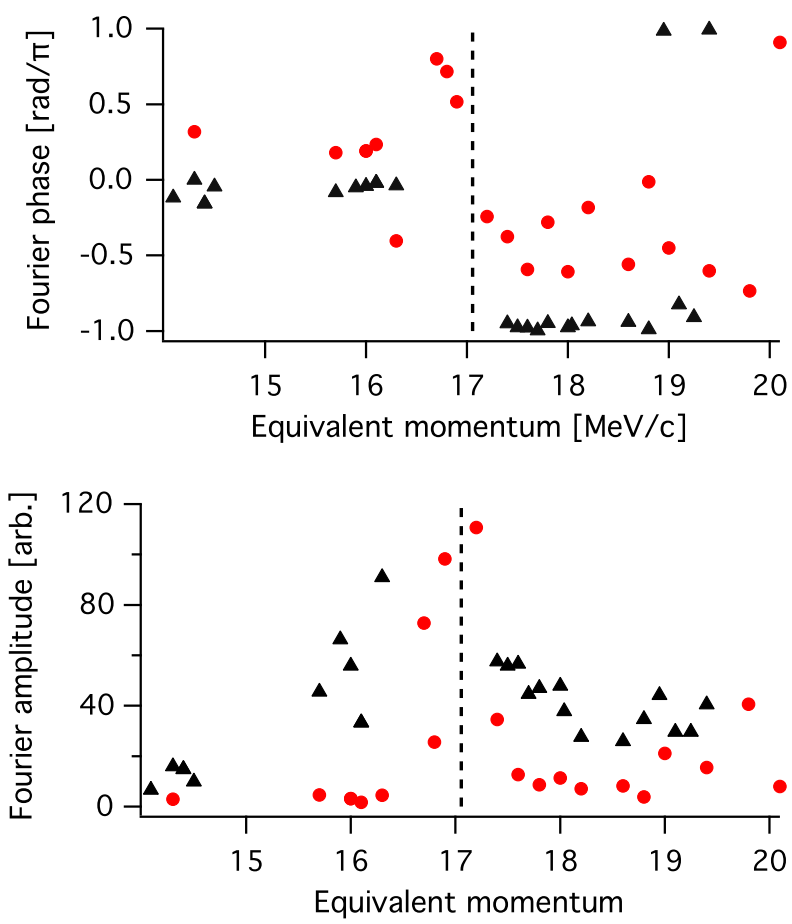

FIG. 11. Harmonic 7 phase (top panel) and amplitude (bottom panel) before (black triangles) and after (red dots) correction obtained from horizontal COD measurements. The vertical line shows the approximate location of integer tune 7 .

errors (e.g., BPM offsets) when the underlying COD is reduced.

\section{DISCUSSION}

In practice, deviations from an ideal linear NS-FFAG may mean that individual harmonics cannot be corrected in isolation. Random errors in the quadrupole gradient, nonlinearity in the magnet field profile (e.g., sextupole), and the finite accuracy of the magnet displacement will result in the excitation of undesired harmonics. The measured response of the closed orbit to the corrector excitation should, in principle, allow errors in the quadrupole gradient to be ascertained (as in the linear optics from closed orbits technique [13]). This information could then be used to reduce the COD further.

The number of installed corrector $N_{C}$ limits the highest harmonic that can be corrected to $N_{C} / 2$. In EMMA, this is an issue in the vertical plane where $N_{C} / 2$ is lower than the highest integer tune in the momentum range. During $10-20 \mathrm{MeV} / c$ acceleration, the beam is coherently excited as it passes through the uncorrectable high integer tunes. However, we propose that by setting the appropriate amplitude and phase at harmonic $N_{C} / 2$ to cancel the distortion, while at the same time correcting lower harmonics as before, the uncorrected distortion can be restricted to the high tune and low momentum region. This will be a topic of future study.
Synchrobetatron effects caused by nonadiabatic acceleration also contribute to the distortion. In EMMA, there is a cavity in every other cell in the ring, apart from in the injection and extraction cells. These "missing cavities" enhance synchrobeta distortion compared to the case where the arrangement is symmetric. Simulations indicate that in EMMA the distortion due to this source is relatively small.

Despite these limitations, the experimental results demonstrate that correcting the principle harmonics, by way of a least-squares correction at multiple momenta, succeeds in reducing the COD over the momentum range. It is of interest to test whether the correction also reduces the accelerated orbit distortion as suggested by simulation. For the case of a particle performing synchrotron oscillations, measurement of the orbit distortion is complicated by the decoherence of betatron oscillations (made more rapid by coupling from the longitudinal motion). However, it can be seen that, apart from in the region of integer tunes, the rms of the accelerated orbit calculated over a moving window is similar to the COD and is also reduced by the correction [14]. For the case of the serpentine channel, acceleration may be completed before the beam decoheres; hence, it may be possible to observe a reduction in distortion as in Fig. 5. Experimental verification of correction in this regime has not been carried out to date.

The lessons learned from correcting the orbit distortion in EMMA should inform future designs of linear nonscaling FFAGs. One major source of orbit distortion, the septum stray field, should be addressed when considering how to carry out injection and extraction. A method to determine the BPM offsets (for example, using beam-based methods) would reduce the uncertainty in the closed orbit measurements.

More fundamentally, knowing how well orbit distortion can be controlled allows an assessment of the feasibility of other proposed nonscaling lattices. As described in Sec. III, the crossing of integer tunes largely drives the accelerated orbit distortion in linear nonscaling FFAGs. Theoretical predictions $[15,16]$ and experiment [17] show that the distortion due to resonance crossing grows with the square root of the crossing speed. It follows that in situations where slow acceleration is inevitable, for example, in the acceleration of protons (for example, in hadron therapy [3]), the amplification factor is large. Previous studies suggested that the orbit distortion could be reduced by at most 50\% [18]. However, by using harmonic correction, there is nothing in principle that limits the correction that can be achieved. In practice, the correction will be limited by the accuracy of measurements of the harmonic components of the COD and the finite accuracy with which the correction can be applied. These practical limits are specific to the technology employed in each accelerator and should be studied for each case individually. 


\section{ACKNOWLEDGMENTS}

The authors thank Alexander Kalinin and Graham Cox for ensuring the successful operation of the EMMA BPM system.

[1] R. J. Abrams et al., arXiv:1112.2853; Report No. BNL96453-2011; Report No. CERN-ATS-2011-216; Report No. FERMILAB-PUB-11-581-APC; Report No. RALTR-2011-018, 2011.

[2] V.S. Morozov, S. A. Bogacz, and D. Trbojevic, in Proceedings of the International Particle Accelerator Conference, Kyoto, Japan (ICR, Kyoto, 2010), p. 3539.

[3] E. Keil, A. M. Sessler, and D. Trbojevic, Phys. Rev. ST Accel. Beams 10, 054701 (2007).

[4] E. C. Aschenauer et al., arXiv:1409.1633.

[5] S. Koscielniak and C. Johnstone, Nucl. Intrum. Methods Phys. Res. Sect. A 523, 25 (2004).

[6] S. Machida et al., Nat. Phys. N8, 243 (2012).

[7] S. Machida et al. (to be published).
[8] R. Barlow et al., Nucl. Instrum. Methods Phys. Res., Sect. A 624, 1 (2010).

[9] I. W. Kirkman et al., in Proceedings of the 3rd International Particle Accelerator Conference, New Orleans, LA (IEEE, Piscataway, NJ, 2012), p. 921.

[10] S. Y. Lee, Accelerator Physics (World Scientific, Singapore, 2004).

[11] F. Méot, Nucl. Instrum. Methods Phys. Res., Sect. A 767, 112 (2014).

[12] C. S. Edmonds, J. Gratus, K. M. Hock, S. Machida, B. D. Muratori, R. G. Torromé, and A. Wolski, Phys. Rev. ST Accel. Beams 17, 054401 (2014).

[13] J. Safranek, Nucl. Instrum. Methods Phys. Res., Sect. A 388, 27 (1997).

[14] J. M. Garland et al. (to be published).

[15] G. Guignard, CERN Report No. 77-10, 1977.

[16] R. Baartman, in Proceedings of the Fixed Field Alternating Gradient Workshop, Vancouver, 2004 (unpublished).

[17] K. Moriya et al. (to be published).

[18] D. J. Kelliher and S. Machida, in Proceedings of the 11th European Particle Accelerator Conference, Genoa, 2008 (EPS-IGA and CERN, Geneva, 2008), p. 3383. 